\title{
DIMENSIONAMENTO E SOBREPOSIÇÃO DE NICHOS DOS PORTUNÍDEOS (DECAPODA, BRACHYURA), NA ENSEADA DA FORTALEZA, UBATUBA, SÃO PAULO, BRASIL
}

\author{
Marcelo Antonio Amaro Pinheiro 1,3 \\ Adilson Fransozo 2, 3 \\ Maria Lúcia Negreiros-Fransozo ${ }^{2,3}$
}

\begin{abstract}
BREADTH AND OVERLAP NICHES OF THE PORTUNID CRABS (DECAPODA, Brachyura), In Fortaleza Bay, Ubatuba, São Paulo, Brazil. The niche breadth of the Portunidae and their overlap on the subtidal sediments of Fortaleza bay, Ubatuba (São Paulo) was analyzed. Samples were made monthly from November/1988 to October/1989, in seven areas of the bay using a shrimp fishery boat equipped with two otter-trawls. Each area was characterized based on environmental factors such as depth, temperature, salinity, dissolved oxygen, organic matter and granulometric composition of the sediment. The number of individuals of each species was registered to each area (resource). Levins's standardized measure (BA) and niche percentage were calculated. Five species of swimming crabs were recorded in this study: Callinectes ornatus Ordway, 1863, Arenaeus cribrarius (Lamarck, 1818), Portunus spinimanus Latreille, 1819, Callinectes danae Smith, 1869 and Portunus spinicarpus (Stimpson, 1871). The widest ecological niche occurred to C. ornatus, present in all sampled areas (generalist species). Such fact can be related to high sediment tolerance when it is compared to $P$. spinimanus (especialist species) which was limited to the areas with coarse granulometric fractions. Highest niche overlap was verified between C. danae and A. cribrarius may be due to greater salinity tolerance of these species. The low occurrence of $P$. spinicarpus and its reduced niche size in Fortaleza Bay are due to association of this species to cold water currents (ACAS) more evidente in smaler depths during summer months. One future evaluation of the portunid diet can be useful to complement informations about this important aspect of the marine ecology.

KEY WORDS. Portunidae, distribution, niche, sediment, swimming crab
\end{abstract}

A área ocupada por determinado organismo na natureza é reflexo da atuação isolada ou conjunta de fatores extrínsecos, sejam eles ambientais ou bióticos. Com relação à fauna bentônica marinha, têm-se verificado que os fatores ambientais mostram maior influência nos padrões de distribuição quando comparados com os bióticos (FoRNERIS 1969).

1) Departamento de Biologia Aplicada, Faculdade de Ciências Agrárias e Veterinárias, Universidade Estadual Paulista. 14870-000 Jaboticabal, São Paulo, Brasil.

2) Departamento de Zoologia, Instituto de Biociências, Universidade Estadual Paulista. 18618-000 Botucatu, São Paulo, Brasil.

3) Centro de Aqüicultura, Universidade Estadual Paulista, Núcleo de Estudos em Biologia Ecologia e Cultivo de Crustáceos. 
Várias espécies de caranguejos e siris seguem determinado padrão de distribuição, principalmente em relação à variações de salinidade, granulometria e teor de matéria orgânica do sedimento onde vivem (FRANSOZO et al. 1992; SANTOS et al. 1994; PINHEIRO et al. 1996).

$\mathrm{Na}$ natureza não são raras as espécies que utilizam os mesmos recursos abióticos ou bióticos. Assim, o compartilhamento parcial ou total de tais áreas de distribuição dependem da tolerância das espécies simpátricas ao recurso utilizado $\mathrm{e}$, conseqüentemente, da competitividade entre elas.

Conforme MAGUIRE (1973), conceitua-se nicho ecológico como a "tolerância e resposta biológica de um indivíduo, de uma população de determinada espécie ou conjunto de espécies às condições ambientais". Portanto, a elucidação de seu dimensionamento e de possíveis sobreposições entre nichos são informações ecológicas de extrema importância.

Os braquiúros não têm sido devidamente abordados na literatura quanto a este aspecto, destacando-se poucos trabalhos como o de ORTMANN (1906) sobre caranguejos de rio. Contribuições neste sentido seriam de extrema importância, particularmente no que se refere aos representantes de Portunidae, que preponderam em número numa comparação a nível de carcinofauna bentônica marinha (FRANSOZO et al. 1992; VANIN 1989)

Este trabalho visa dimensionar o nicho ecológico dos portunídeos do sedimento sublitoral da Enseada da Fortaleza, Ubatuba, São Paulo, e suas prováveis sobreposições, baseando-se em seus aspectos distribucionais e bio-ecológicos.

\section{MATERIAL E MÉTODOS}

Foram realizadas coletas mensais no período de novembro/1988 a outubro/1989 em sete áreas de amostragem localizadas na Enseada da Fortaleza, Ubatuba, São Paulo (Fig. 1). A fauna bentônica de cada uma foi amostrada por arrastos de $1 \mathrm{~km}$ de extensão com redes do tipo otter-trawl tracionadas por um barco camaroeiro.

No ponto médio de cada arrasto a profundidade foi mensurada por sondagem manual. Além disso, foram também coletadas amostras da água de fundo com uma garrafa de Nansen e do sedimento com um pegador de van Veen. Tais amostras serviram para a mensuração dos parâmetros ambientais de cada área durante o período de estudo, conforme segue: temperatura da água (termômetro); teor de oxigênio dissolvido na água (técnica de Winkler); salinidade (refratômetro óptico); composição granulométrica do sedimento (peneiramento diferencial em sete frações granulométricas); e teor de matéria orgânica do sedimento (pesagem diferencial após três horas em mufla a $500^{\circ} \mathrm{C}$ ). Tais resultados foram utilizados para a caracterização abiótica das áreas de amostragem, podendo ser consultados no trabalho de NEGREIROS-FRANSOZO et al. (1991).

Após triagem prévia, os exemplares de Portunidae foram acondicionados em sacos plásticos devidamente etiquetados e mantidos sob congelamento até seu manuseio. Em laboratório foram identificados segundo WiLLIAMS (1984), separados por espécie e posteriormente contados. 


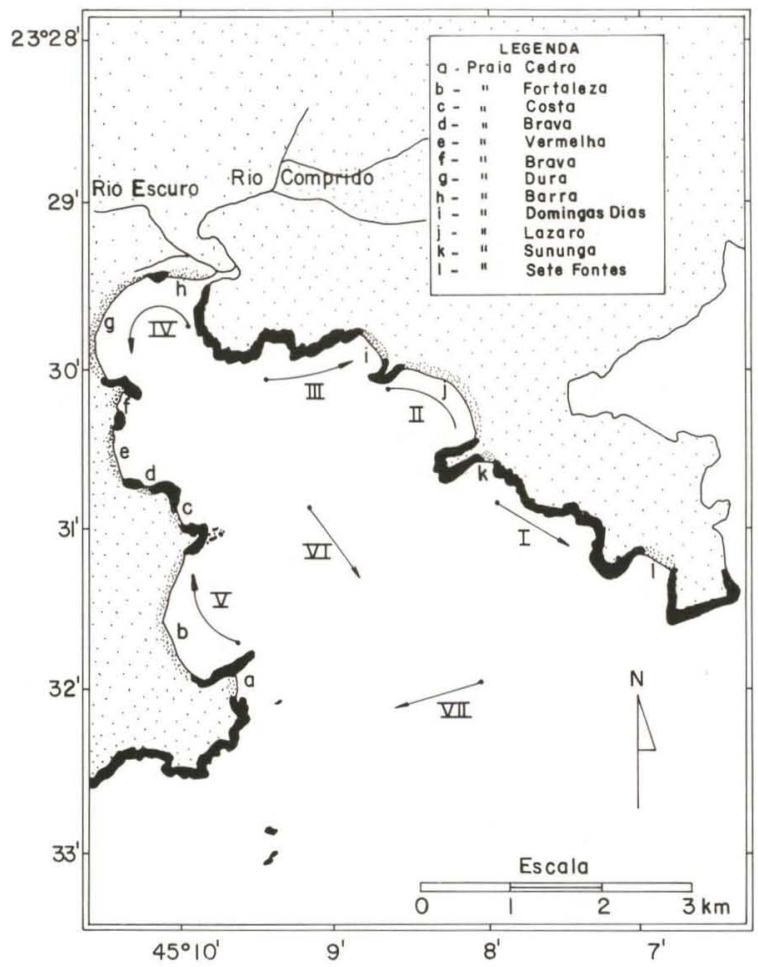

Fig. 1. Mapa da Enseada da Fortaleza, Ubatuba, São Paulo, mostrando a localização das sete áreas de coleta amostradas no período de novembro/1988 a outubro/1989.

De acordo com KREBS (1989) a amplitude do nicho pode ser mensurada pela distribuição dos indivíduos de uma espécie dentro de um conjunto de recursos. No presente trabalho conceituou-se como recurso cada uma das sete áreas onde os animais foram coletados (unidades de amostragem artificial), baseando-se nas diferenças físico-químicas a elas peculiares (KREBS 1989: 372). Tais dados serviram para a elaboração de uma matriz de recursos, onde as espécies são dispostas em linhas e os recursos em colunas (COLWELL \& FUTUYMA 1971). A partir dela calculou-se a amplitude do nicho de cada espécie pela medida de Levins padronizada (BA) (KREBS 1989: 374) e o percentual de sobreposição existente entre eles (KREBS 1989: 381), conforme segue:

$$
B_{A}=\frac{B-1}{n-1}
$$

onde: $\left(\mathrm{B}_{\mathrm{A}}\right)$ medida de Levins padronizada (varia de 0 a 1$) ;\left(\mathrm{B}=1 / \Sigma \mathrm{p}_{\mathrm{j}}{ }^{2}\right)$ medida de Levins (amplitude do nicho); $\left(\mathrm{p}_{\mathrm{j}}=\mathrm{N}_{\mathrm{j}} / \mathrm{y}\right)$ proporção de indivíduos utilizando o recurso “j”; $\left(\mathrm{N}_{\mathrm{j}}\right)$ número de indivíduos encontrados utilizando o recurso " $\mathrm{j}$ ”; $\left(\mathrm{Y}=\Sigma \mathrm{N}_{\mathrm{j}}\right)$ número total de indivíduos amostrados; (n) número total de recursos. 


$$
P_{j k}=\left[\sum\left(\text { mínimo } p_{i j}, p_{i k}\right)\right] \cdot 100
$$

onde: $\left(\mathrm{P}_{\mathrm{jk}}\right)$ porcentagem de sobreposição existente entre o nicho das espécies “j”” e " $k$ "; ( $\left.p_{i j}, p_{i k}\right)$ proporção do recurso " $i$ " em relação ao total dos recursos utilizados pela espécies "j" e pela espécie " $k$ "; (n) número total de recursos.

\section{RESULTADOS}

As áreas de amostragem apresentaram-se bem heterogêneas em relação a atuação dos parâmetros abióticos, podendo ser descritas como segue:

Área I. Área de mar aberto; profundidade média relativamente acentuada $(11,17 \mathrm{~m})$; sedimento com predomínio das frações areia média, muito fina e silte+argila; Área II. Área de mar fechado; elevado teor médio de matéria orgânica $(6,65 \%)$; sedimento com certa homogeneidade entre as frações granulométricas; águas pouco competentes;

Área III. Área de mar aberto; reduzido teor médio de matéria orgânica (2,32\%); sedimento composto principalmente pelas frações areia muito fina e silte+argila;

Área IV. Área de mar aberto; reduzido teor de matéria orgânica (1,84\%); baixa profundidade $(4,42 \mathrm{~m})$; sedimento com predomínio de areia muito fina e influência de águas estuarinas;

Área V. Área de mar fechado; sedimento composto principalmente por areia fina e muito fina e águas pouco competentes;

Área VI. Área de mar aberto; profundidade média acentuada $(11,08 \mathrm{~m})$; sedimento composto principalmente por areia muito fina e silte+argila;

Área VII. Área de mar aberto; profundidade média acentuada $(13,33 \mathrm{~m})$; sedimento composto principalmente por areia muito fina e silte+argila.

De acordo com NEGREIROS-FRANSOZO et al. (1991) as áreas amostradas na Enseada da Fortaleza apresentaram certa homogeneidade quanto ao teor de oxigênio dissolvido, temperatura e salinidade, mostrando as menores variações quando comparados com os demais fatores ambientais estudados.

Durante o período de estudos foram registradas cinco espécies de siris: Callinectes ornatus Ordway, 1863 ( $\mathrm{N}=2.217)$; Arenaeus cribrarius (Lamarck, 1818) (N=245); Portunus spinimanus Latreille, 1819 ( $\mathrm{N}=126)$; Callinectes danae Smith, 1869 (N=102) e Portunus spinicarpus (Stimpson, 1871) (N=2). As espécies $P$. spinimanus e $P$. spinicarpus apresentaram-se distribuídas somente em duas das áreas amostradas, diferente das demais que ocorreram indistintamente em todas as sete áreas.

O nicho de maior amplitude foi do siri $C$. ornatus, seguido pelo de $A$. cribrarius, $C$. danae, $P$. spinicarpus e $P$. spinimanus (Tab. I). Não foi verificada qualquer sobreposição dos nichos de $P$. spinicarpus e $P$. spinimanus, apesar disto ocorrer com o nicho das demais espécies (Tab. II). Os menores percentuais de sobreposição de nichos ocorreram entre $A$. cribrarius e as espécies do gênero Portunus $(5,71 \%)$, enquanto o maior foi verificado entre $A$. cribrarius e $C$. danae $(59,52 \%)$. 
Em função da composição granulométrica do sedimento, pode-se observar a ocorrência de cada uma das cinco espécies de portunídeos coletadas na Enseada da Fortaleza (Fig. 2).

Tabela I. Matriz de recursos e dimensionamento do nicho ecológico das espécies de Portunidae coletadas na Enseada da Fortaleza, Ubatuba, São Paulo, durante o período de novembro/1988 a outubro/1989. ( $\left.\mathrm{B}_{\mathrm{A}}\right)$ Medida de Levins padronizada, $(\mathrm{N})$ número de individuos, conforme KREBS (1989: 374).

\begin{tabular}{|c|c|c|c|c|c|c|c|c|c|}
\hline \multirow{2}{*}{ Espécies } & \multirow{2}{*}{$\begin{array}{c}N \\
(\%)\end{array}$} & \multicolumn{7}{|c|}{ Áreas de amostragem } & \multirow{2}{*}{$\mathrm{B}_{\mathrm{A}}$} \\
\hline & & 1 & ॥ & III & IV & V & VI & VII & \\
\hline \multirow[t]{2}{*}{ Callinectes ornatus (Ordway, 1863) } & $\mathrm{N}$ & 347 & 891 & 280 & 71 & 207 & 193 & 228 & 0,56 \\
\hline & $\%$ & 15,65 & 40,19 & 12,63 & 3,20 & 9,34 & 8,71 & 10,28 & \\
\hline \multirow[t]{2}{*}{ Arenaeus cribrarius (Lamarck, 1818) } & $\mathrm{N}$ & 39 & 4 & 103 & 75 & 10 & 4 & 10 & 0,39 \\
\hline & $\%$ & 15,92 & 1,63 & 42,05 & 30,61 & 4,08 & 1,63 & 4,08 & \\
\hline \multirow[t]{2}{*}{ Callinectes danae Smith, 1869} & $\mathrm{~N}$ & 4 & 4 & 16 & 60 & 2 & 10 & 6 & 0,26 \\
\hline & $\%$ & 3,92 & 3,92 & 15,69 & 58,83 & 1,96 & 9,80 & 5,88 & \\
\hline \multirow[t]{2}{*}{ Portunus spinicarpus (Stimpson, 1871) } & $\mathrm{N}$ & - & - & - & - & - & 1 & 1 & 0,17 \\
\hline & $\%$ & - & - & - & - & - & 50,00 & 50,00 & \\
\hline \multirow[t]{2}{*}{ Portunus spinimanus Latreille, 1819} & $N$ & - & 98 & - & - & 28 & - & - & 0,09 \\
\hline & $\%$ & - & 77,78 & - & - & 22,22 & - & - & \\
\hline
\end{tabular}

Tabela II. Percentual de sobreposição entre os nichos ecológicos dos portunídeos coletados nas sete áreas de coleta da Enseada da Fortaleza, Ubatuba, São Paulo, no período de novembro/1988 a outubro/1989.

\begin{tabular}{lcccc}
\hline \multicolumn{1}{c}{ Espécies } & Arenaeus cribrarius & Callinectes ornatus & Callinectes danae & Portunus spinicarpus \\
\hline Callinectes ornatus & 42,90 & - & - & - \\
Callinectes danae & 59,52 & 40,22 & - & - \\
Portunus spinicarpus & 5,71 & 18,99 & 15,68 & - \\
Portunus spinimanus & 5,71 & 48,53 & 5,88 & 0,00 \\
\hline
\end{tabular}

\section{DISCUSSÃO E CONCLUSÕES}

Os animais marinhos encontram-se sujeitos a atuação de diversos fatores ambientais que podem variar a nível espacial, sazonal ou temporalmente. Os parâmetros ambientais de maior variação podem limitar a sobrevivência e distribuição de uma espécie em função de sua tolerância. As espécies mais tolerantes mostram maior sucesso na conquista de ambientes heterogêneos sendo denominadas generalistas, ao contrário das especialistas, que devido a sua menor tolerância a certos recursos mostram-se mais localizadas ou endêmicas.

Conforme Melo (comunicação pessoal) a distribuição dos braquiúros mostra padrões de associação com certos parâmetros ambientais, particularmente os edáficos. As espécies simpátricas apresentam afinidade por um ou mais recursos e, conseqüentemente, maior sobreposição entre seus nichos. Nestas áreas pode ocorrer a exclusão competitiva de uma das espécies (HUTCHINSON 1981) ou a partição ambiental. 


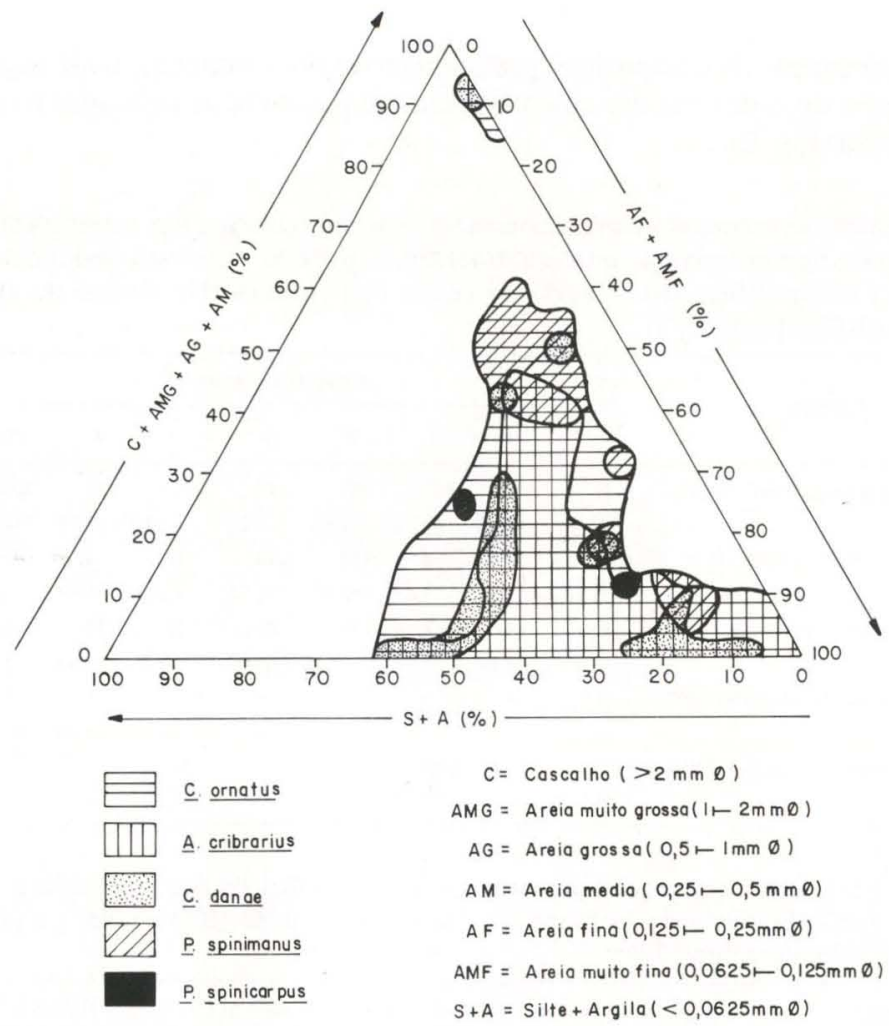

Fig. 2. Diagrama de ocorrência dos portunídeos da Enseada da Fortaleza, Ubatuba, São Paulo, baseado nos percentuais granulométricos do sedimento marinho.

Os animais de maior atividade locomotora minimizam o estresse causado por recursos ambientais inadequados valendo-se de comportamentos peculiares de fuga (ODUM 1988). O achatamento dorso-ventral do último par de pereiópodos dos portunídeos é uma adaptação que possibilita a natação por curtas distâncias, favorecendo, assim, a fuga rápida do estímulo agressor. Além disso, estes animais frequentemente se enterram no sedimento protegendo-se de predadores ou mesmo para facilitar a captura de presas ágeis como os peixes (SCHÄFER 1954 apud SCHÖNE 1961).

Apesar dos portunídeos poderem ingerir o sedimento como um todo e extrair sua matéria orgânica, tais animais são basicamente carnívos, com dieta composta por animais predados ou já em decomposição (WARNER 1977). Isto favorece uma sobreposição trófica com outras espécies simpátricas, principalmente as congenéricas ou de mesma equivalência ecológica, que muitas vezes se tornam especializadas na extração de determinado recurso alimentar. Como exemplo pode-se citar os artigos de WADE (1967) e LEBER (1982) que relatam a predação de $A$. cribrarius sobre espécies dos gêneros Emerita Scopoli, 1777 e Donax Linnaeus, 1758, na zona de arrebentação de maré de praias arenosas. 
O nicho ocupado por determinada espécie na natureza mostra melhor relação com os recursos ambientais do que com as interações bióticas. C. ornatus caracterizou-se como o portunídeo mais generalista, possivelmente devido a sua maior tolerância em relação à composição granulométrica do sedimento (espécie euritópica). As demais espécies foram mais especialistas fazendo sempre parte do nicho ocupado por $C$. ornatus, com o qual mostraram a maior sobreposição.

A maior abundância de $A$. cribrarius, $C$. ornatus e $C$. danae na Enseada da Fortaleza está associada à presença de um sedimento constituído predominantemente pelas frações arenosas mais finas. Por outro lado, a ocorrência de espécies estenotópicas como $P$. spinimanus foi favorecida pela presença de frações arenosas mais grossas e águas de menor competência, características das áreas II e V da Enseada da Fortaleza (SANTOS et al. 1994).

O nicho ecológico de A. cribrarius foi o segundo em tamanho, mostrando elevado percentual de sobreposição com $C$. danae possivelmente devido a eurihalinidade destas espécies. Estas são encontradas com maior frequiência em áreas rasas, com certa influência estuarina e predomínio de sedimento arenoso mais fino (PINHEIRO et al. 1996; FRANSOZO et al. 1992).

A pequena representatividade e menor tamanho de nicho verificadas para $P$. spinicarpus deve-se à sua associação com correntes de águas frias (ACAS), que apresentam maior penetração junto à plataforma continental do litoral norte paulista nos meses de verão (SARTOR 1989; VANIN 1989). Por este motivo, tal espécie apresenta-se mais abundante em maiores profundidades $(>50 \mathrm{~m})$, explicando sua inexpressividade na Enseada da Fortaleza cuja profundidade média foi de nove metros (NEGREIROS-FRANSOZO et al. 1991).

Uma futura avaliação da dieta destes portunídeos, bem como a afinidade por cada item alimentar, poderão corroborar e complementar tais afirmações, elucidando completamente este importante aspecto da ecologia de crustáceos marinhos.

\section{REFERÊNCIAS BIBLIOGRÁFICAS}

ColweLl, R.K. \& D.J. FutUYMA. 1971. On the measurement of niche breadth and overlap. Ecology 52: 567-576.

FoRNERIS, L. 1969. Fauna bentônica da Baía do Flamengo, Ubatuba. Aspectos ecológicos. Tese de Livre-Docência, não publicada, Faculdade de Filosofia, Ciências e Letras, Universidade de São Paulo, São Paulo, 215p.

Fransozo, A.; M.L. Negreiros-Fransozo; F.L.M. Mantelatto; M.A.A. PINHEIRO \& S. SANTOS. 1992. Composição e distribuição dos Brachyura (Crustacea, Decapoda) do substrato não consolidado da Enseada da Fortaleza, Ubatuba (SP). Rev. Brasil. Biol. 52 (4): 667-675.

HUTCHINSON, G.E. 1981. Introducción a la ecologia de poblaciones. Barcelona, Editorial Blume, 492p.

KREBS, C.J. 1989. Ecological methodology. New York, Harper \& Row Publishers, $645 \mathrm{p}$.

LEBER, K.M. 1982. Seasonality of macroinvertebrates on a temperate, high wave energy sandy beach. Bull. Mar. Sci. 32 (1): 86-98. 
MAGUIRE JR., B. 1973. Niche response structure and the analytical potentials of its relationship to the habitat. Amer. Nat. 107 (954): 213-246.

Negreiros-Fransozo, M.L.; A. Fransozo; M.A.A. PinHeiro; F.L.M. MANTELATTO \& S. SANTOS. 1991. Caracterização física e química da Enseada da Fortaleza, Ubatuba, SP. Rev. Bras. Geocienc. 21 (3): 114-120.

ODuM, E. P. 1988. Ecologia. Rio de Janeiro, Editora Guanabara S.A., 434p.

ORTMANN, A.E. 1906. Facts and theories in evolution. Science 23: 947-952.

Pinheiro, M.A.A.; A. Fransozo \& M.L. Negreiros-Fransozo. 1996. Distribution patterns of Arenaeus cribrarius (Lamarck, 1818) (Crustacea, Brachyura, Portunidae), in Fortaleza Bay, Ubatuba (SP), Brazil. Rev. Brasil. Biol. 56 (4): 705-716.

Santos, S.; M.L. Negreiros-Fransozo \& A. Fransozo. 1994. The distribution of the swimming crab Portunus spinimanus Latreille, 1819 (Crustacea, Brachyura, Portunidae) in Fortaleza Bay, Ubatuba, SP, Brazil. Atlântica 16: 125141.

SARTOR, S.M. 1989. Composição e distribuição dos Brachyura (Crustacea, Decapoda), no litoral norte do Estado de São Paulo. Tese de Doutorado, não publicada, Instituto Oceanográfico, Universidade de São Paulo (IO/USP), São Paulo, 197p.

SCHÄFER, W. 1954. Form and funktion der Brachyurenschere. Abhandkl. Senckenberg. naturf. Ges. 489: 1-66.

SCHÖNE, H. 1961. Complex behavior, p.465-520. In: T.H. WATERMAN. The phisiology of Crustacea. Sense organs, integration and behaviour. New York, Academic Press, vol. 2, 681p.

VANIN, A.M.S.P. 1989. Estrutura e dinâmica da megafauna bêntica na plataforma continental da região norte do Estado de São Paulo, Brasil. Tese de Livre-Docência, não publicada, Instituto Oceanográfico, Universidade de São Paulo, São Paulo, 215p.

WADE, B.A. 1967. Studies on the biology of the west indian beach clam, Donax denticulatus Linné. 1 - Ecology. Bull. Mar. Sci. 17 (1): 149-174.

WARNER, G.F. 1977. The biology of crabs. New York, Elek Science London, 202p. Williams, A.B. 1984. Shrimps, lobsters and crabs of the Atlantic Coast of the eastern United States, Maine to Florida. Washington, Smithsonian Institution Press, XVIII+550p.

Recebido em 17.VI.1996; aceito em 18.VI.1997. 